\title{
INATIVIDADE FÍSICA EM CRIANÇAS: UMA REVISÃO SISTEMÁTICA DE ESTUDOS REALIZADOS NO BRASIL
}

\author{
PHYSICAL INACTIVITY IN CHILDREN: A SYSTEMATIC REVIEW OF STUDIES \\ CONDUCTED IN BRAZIL
}

\author{
Daiane Aragão ${ }^{a^{*}}$, Camilo Luis Monteiro Lourenço ${ }^{b^{* *}}$, Thiago Ferreira de Sousa ${ }^{* *}$ \\ adai-aragao@hotmail.com, bcamilomonteiro@outlook.com, ctfsousa_thiago@yahoo.com.br \\ *Universidade Estadual de Santa Cruz - Ilhéus (BA), Brasil \\ **Universidade Federal do Triângulo Mineiro - Uberaba (MG), Brasil
}

Data de recebimento do artigo: 30/07/2014

Data de aceite do artigo: 06/02/2015

\section{RESUMO}

Introdução: $\mathrm{O}$ processo de urbanização, os avanços tecnológicos, e as alteraçôes econômicas, políticas e sociais, influenciaram os níveis de atividades físicas das pessoas, levando-as ao sedentarismo, que pode acarretar riscos à saúde em todas as faixas etárias. Objetivo: Descrever a prevalência de inatividade física em crianças brasileiras, por meio de uma revisão sistemática da literatura. Materiais e métodos: Foi realizada uma revisão sistemática dos artigos publicados na base de dados Bireme em agosto e setembro de 2013, utilizando na busca os seguintes termos: "atividade física"; "atividade motora"; "exercício físico"; "inatividade física"; "sedentarismo"; "gasto energético"; "aptidão física"; "fator de risco"; "esportes"; "fator"; "conduta de saúde"; "estilo de vida"; "saúde"; "educação física”; "criança”; "escolar"; "infância"; "juventude"; "saúde da criança"; "infantil"; "Brasil" or "brasileiro". Foram incluídos artigos publicados de 2004 a 2013. Resultados: A busca inicial resultou em 58 artigos, e ao final foram selecionados, para compor a revisão, 7 manuscritos, sendo 2 obtidos nas listas de referências. A prevalência de inatividade física entre os estudos variou de 14,2 a 73,9\%. O delineamento transversal foi predominante entre os estudos, e apenas 1 deles adotou o tipo coorte. Do total, três publicaçôes foram provenientes da regiáo Sudeste. O questionário foi o principal instrumento para mensurar o nível de atividade física em crianças. Conclusóes: A prevalência de inatividade física verificada nos estudos com crianças brasileiras foi elevada. Contudo, é necessária a realização de mais estudos, principalmente em diferentes regiōes brasileiras.

Palavras-chave: Atividade motora; infância; revisão por pares; brasileiros.

\section{ABSTRACT}

Introduction: The urbanization process, technological advances, economic, political and social changes influence the physical activity levels in persons, and the physical inactivity can promote risks to health in all ages. Objective: The purpose of this study was to describe the prevalence of physical inactivity among Brazilian children, by a systematic review. Materials and Methods: A systematic review of articles published in Bireme database was carried out. The search was conducted between August and September of 2013 and used the combination of terms: "physical activity"; "motor activity"; "exercise"; "physical inactivity"; "sedentary"; "energy expenditure"; "physical fitness"; "risk factor"; "sports"; "factor"; "health behavior"; "lifestyle"; "health"; "physical education"; "child"; "school”; "childhood"; "youth"; "child health"; "Brazil" and "Brazilian". Only the articles published from 2004 to 2013 were included. Results: The initial search resulted in 58 articles. Three were classified as repeated, and in the end, eight manuscripts were selected for review, and two were obtained from the list of references. A variation in the prevalence of physical inactivity was from $14.2 \%$ to $73.9 \%$. The cross-sectional design was prevalent among the studies. Of all of publications, three were from the southeast region. The questionnaire was the main instrument to measure the level of physical activity in children. Conclusions: The prevalence of physical inactivity in studies with Brazilian children was high. However, it's necessary to carry out more studies in different regions.

Keywords: Physical activity; childhood; peer review; Brazilian. 


\section{Introdução}

A prática regular de atividade física e sua relevância para a saúde e qualidade de vida tem sido tema de discussão em diversos estudos, bem como a associação de inatividade física e/ou sedentarismo com as doenças cardiovasculares, cardiorrespiratórias, obesidade e outras comorbidades que acometem as pessoas de todas as faixas etárias ${ }^{1-6}$. Apesar de oferecer benefícios à saúde e evitar o acometimento de muitas doenças, estudos demonstram que a prática de atividade física não tem sido incluída à rotina de grande parte da população brasileira, tanto em adolescentes ${ }^{3}$ quanto em adultos ${ }^{7,8}$.

Estudos de revisão sistemática demonstram o quadro de prevalência de inatividade física em diferentes grupos populacionais ${ }^{3,7-9}$. Hallal et al. $^{7}$ constataram que a prevalência de inatividade física oscilou entre 26,7 e $78,2 \%$, considerando a atividade física em dois ou mais domínios. Quando considerada apenas a atividade física realizada no tempo de lazer, a prevalência variou de 55,3 a $96,7 \%$. Sousa ${ }^{9}$ observou variação na prevalência de 0 a $78,9 \%$ entre estudantes universitários brasileiros.

Em relação às crianças, não foram observados estudos de revisão sistemática sobre os baixos níveis de atividade física. Nessa fase da vida, recomenda-se a prática de, no mínimo, 60 minutos diários de atividades físicas, moderadas ou vigorosas ${ }^{10}$. Deve-se realizar, na maior parte desse tempo, atividades aeróbias. Em pelo menos três dias da semana, essas atividades devem ser vigorosas, além de recomendar-se, nesses três dias, a prática de força e resistência musculares. Compondo, assim, o tempo recomendado de exercícios físicos, que trazem fortalecimento e crescimento ósseo ${ }^{10}$.

Um estudo realizado em Florianópolis (SC) observou a prevalência de crianças fisicamente ativas em $45,1 \%$ para alunos da rede privada, e $42,6 \%$ da rede pública 5 . Outro estudo conduzido com crianças de 6 a 10 anos verificou que a prevalência de inatividade física foi de $36,2 \%$ para meninos e de $56,5 \%$ para meninas ${ }^{11}$. Ou seja, mesmo em crianças, a prevalência de baixo nível de atividade física é elevada. Considerando a relevância da prática de atividade física, e a necessidade de levantamento de informações que contribuam para a promoção regular dessa conduta de vida, o objetivo desse estudo foi descrever a prevalência de inatividade física em crianças brasileiras, por meio de uma revisão sistemática da literatura.

\section{Materiais e métodos}

Foi realizada uma revisão sistemática dos artigos publicados de 2004 a 2013, com crianças de 5 a 11 anos de idade. A busca foi realizada na base de dados Bireme, e posteriormente, na lista de referências dos artigos selecionados na última fase do processo de revisão.

Para a realização das buscas, foram considerados os seguintes descritores: "atividade física"; "atividade motora"; "exercício físico"; "inatividade física”; "sedentarismo"; "gasto energético"; "aptidão física"; "fator de risco"; "esportes"; "fator"; "conduta de saúde"; "estilo de vida"; "saúde"; "educaçâo física"; que foram combinados com outros descritores: "criança"; "escolar"; "infância"; "juventude"; "saúde da criança”; "infantil"; além de "Brasil" e "brasileiro". Recorreu-se aos operadores lógicos "or" e "and" para combinaçáo dos descritores/termos utilizados, visando à localização das publicaçóes.

O período de busca foi realizado em agosto e setembro de 2013. Um dos pesquisadores realizou a busca, e, em caso de dúvida da inclusão de um manuscrito, recorreu-se a um segundo pesquisador. Os critérios de inclusão estabelecidos foram: estudos sobre a atividade física; utilização da medida de um ou mais domínios da atividade física (lazer, deslocamentos, atividades domésticas e ocupacionais), sendo a mesma variável de desfecho isolada ou junta; línguas de publicação: português, inglês e espanhol; estudos realizados no Brasil. Optouse por excluir as teses, dissertaçóes, monografias, documentários, resumos de anais, meta-análises, capítulos de livros, revisão de literatura, revisão sistemática, estudo de validação de instrumento, estudos experimentais e estudos de mensuração de aptidão física.

O processo de seleção das publicaçôes compreendeu três etapas. A primeira consistiu na leitura dos títulos dos artigos que foram encontrados na primeira busca. $\mathrm{Na}$ segunda, foi realizada a leitura dos resumos, e quando o resumo não apresentava informaçóes suficientes para tomada de decisão, o artigo era mantido para a etapa seguinte do processo de revisão. $\mathrm{Na}$ última etapa, os trabalhos foram analisados por meio da leitura na íntegra e inclusão de outros artigos que estivessem nas respectivas referências, desde que fossem capazes de atender aos critérios de seleção.

Dos artigos selecionados, foram descritas as seguintes informaçóes: prevalência de inatividade física, por sexo ou não; medida empregada para mensurar a atividade física; domínios da atividade física analisados; definição operacional da inatividade física; estado brasileiro de realização da pesquisa; tipo de estudo adotado; faixa etária das crianças; processo de seleção e tamanho da amostra; e sexo dos participantes.

\section{Resultados}

Foram encontrados 58 trabalhos para leitura dos títulos. Nessa etapa, 17 trabalhos foram excluídos, três 
por serem repetidos e 14 por náo atenderem aos critérios de seleção previamente estabelecidos, restando 41 . $\mathrm{Na}$ segunda etapa, dos 41 artigos, 35 foram excluídos, perfazendo seis para leitura na íntegra. Após a leitura, cinco foram considerados pertencentes à revisão, e incluídos outros dois trabalhos que constavam nas referências. Por fim, sete artigos foram selecionados ${ }^{11-17}$. A Figura 1 apresenta a descrição das etapas desse estudo.

A Figura 2 apresenta o número de publicaçóes sobre inatividade física em crianças brasileiras por ano de publicação. Observou-se que não houve avanço considerável na quantidade de publicaçóes, com aumento em 2006 e 2011.

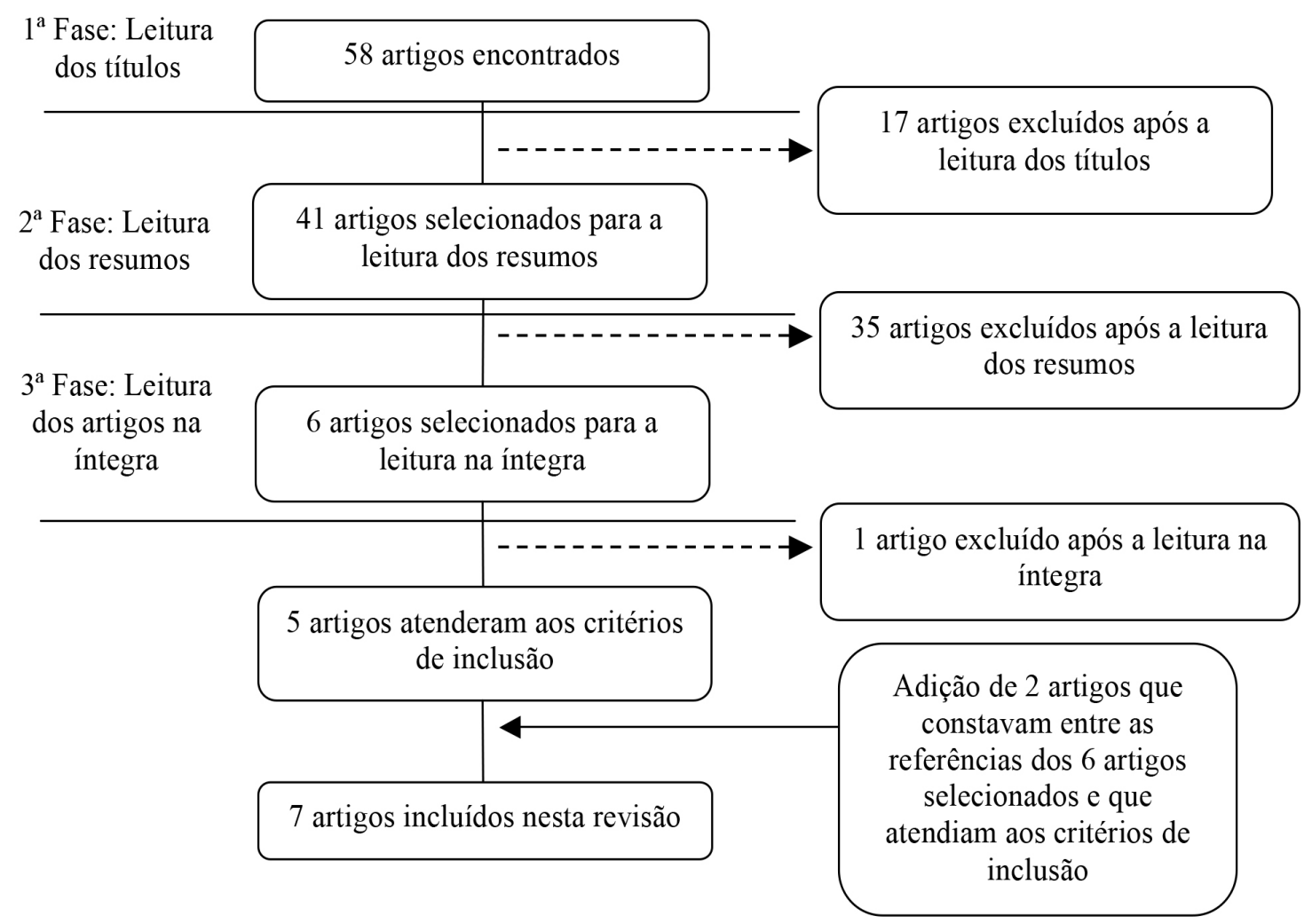

Figura 1: Processo de seleção dos artigos publicados sobre inatividade física em crianças brasileiras.

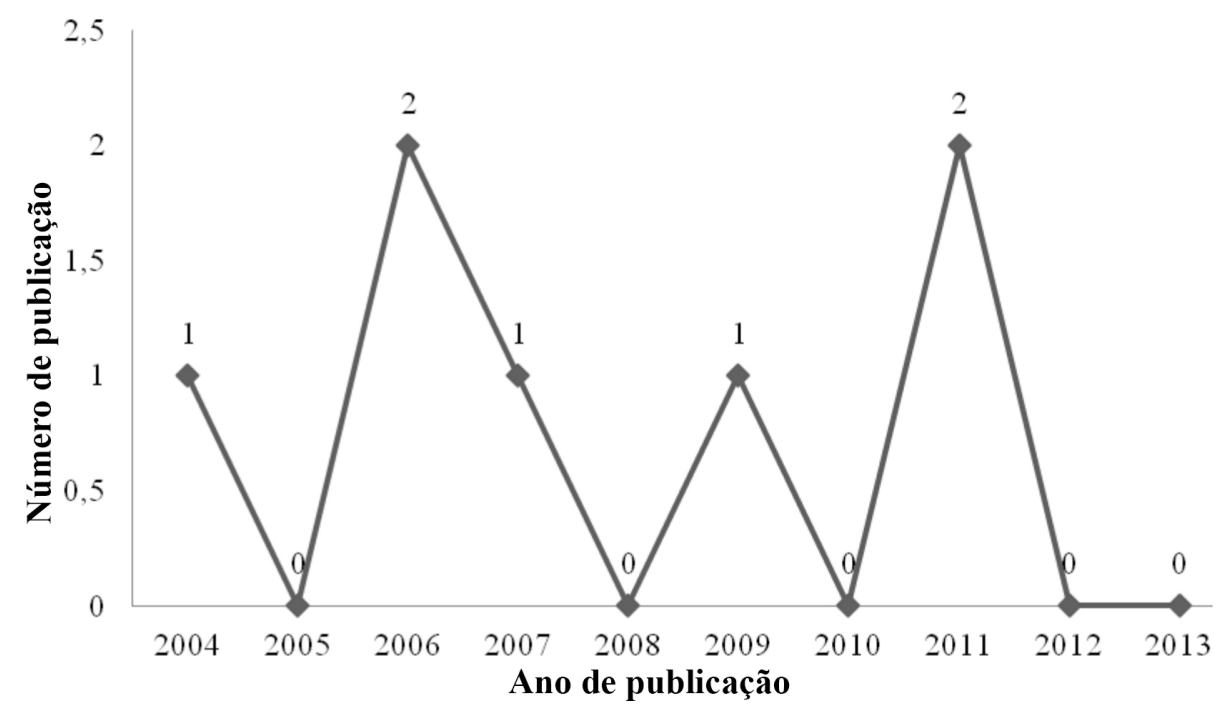

Figura 2: Número de publicações sobre inatividade física em crianças brasileiras por ano. 
$\mathrm{Na}$ Tabela 1 são apresentadas as informaçóes sobre as características das publicações incluídas nessa revisão. $\mathrm{Na}$ maioria dos estudos, o delineamento da pesquisa foi transversal, e um deles foi de coorte ${ }^{16}$. Quanto ao tamanho da amostra, em apenas uma publicação foi observada quantidade inferior a 100 crianças $^{15}$. Nos demais, houve variação de $100^{11}$ a $2.232^{12}$ crianças. Todos incluíram o sexo masculino e feminino, e observou-se, inclusive, que em todos os estudos houve equilíbrio com o número de meninos e meninas (dados não apresentados).

Em relação aos estados onde os estudos foram desenvolvidos (Tabela 1), destacou-se dois do Rio de Janeiro $^{14,16}$, sendo os demais estudos distribuídos entre São Paulo ${ }^{17}$, Santa Catarina ${ }^{12}$, Distrito Federal ${ }^{11}$, Mato Grosso do Sul ${ }^{13}$ e Pernambuco ${ }^{15}$.

Tabela 1: Característica das publicações sobre crianças brasileiras incluídas na revisão.

\begin{tabular}{cccccc}
\hline 10 $^{\circ}$ Autor & Tipo do estudo & Participantes (amostra) & Idade & Sexo & Estado brasileiro \\
\hline Giugliano $^{11}$ & Transversal & 100 & 6 a 10 anos & Masculino e feminino & Distrito Federal \\
Assis $^{12}$ & Transversal & 2.232 & 7 a 9 anos & Masculino e feminino & Santa Catarina \\
Baruki $^{13}$ & Transversal & 403 & 7 a 10 anos & Masculino e feminino & Mato Grosso do Sul \\
Gama $^{14}$ & Transversal & 356 & 5 a 9 anos & Masculino e feminino & Rio de Janeiro \\
Siqueira $^{15}$ & Transversal & 86 & 5 a 9 anos & Masculino e feminino & Pernambuco \\
Gama $^{16}$ & Coorte & $356^{\mathrm{a}}$ & 5 a 9 anos & Masculino e feminino & Rio de Janeiro \\
Mazaro $^{17}$ & Transversal & $331^{\text {b }}$ & 7 a 11 anos & Masculino e feminino & São Paulo \\
\hline
\end{tabular}

$\mathrm{NA}=$ Não apresenta; $\mathrm{a}=$ baseline $; \mathrm{b}=$ follow- $u$.

A Tabela 2 apresenta a definiçáo operacional e a prevalência de inatividade física geral e por sexo. As prevalências de inatividade física variaram de $14,2^{12}$ a $73,9 \%{ }^{15}$, e em um estudo ${ }^{12}$ foi descrita a prevalência de inatividade física específica para o domínio do deslocamento e do lazer. Em todos os artigos foram descritas as prevalências de inatividade física por sexo. Com relaçáo às definiçóes operacionais empregadas nos estudos, observou-se divergência e ausência de melhor detalhamento das informações mensuradas.

Tabela 2: Características dos estudos com crianças brasileiras de acordo com a definição operacional e a prevalência de inatividade física geral e por sexo.

\begin{tabular}{|c|c|c|c|c|}
\hline $1^{\circ}$ Autor & Definiçáo operacional da inatividade física & Domínio da AF & $\% *$ & $\% * *$ \\
\hline Giugliano $^{11}$ & NA & Todos & NA & NA \\
\hline Assis $^{12}$ & $\begin{array}{l}\text { Percepção dos parentes em relaçáo ao deslocamento inativo de casa para a escola } \\
\text { (carro, ônibus ou motocicleta); não praticar esportes fora da escola; e a avaliação } \\
\text { do nível de atividade física como não muito ativo e inativo. }\end{array}$ & $\begin{array}{l}\text { Deslocamento } \\
\text { Lazer } \\
\text { Todos }\end{array}$ & $\begin{array}{l}59,6 \\
64,3 \\
14,2\end{array}$ & NA \\
\hline Baruki $^{13}$ & NA & Todos & NA & NA \\
\hline Gama $^{14}$ & $\begin{array}{c}\text { Praticar }<60 \text { minutos de atividade física por dia, considerando } 6 \text { meses } \\
\text { anteriores. }\end{array}$ & NA & 38,2 & NA \\
\hline Siqueira $^{15}$ & $\begin{array}{l}\text { Não praticar atividade física ou apresentar níveis inferiores ao nível moderada- } \\
\text { mente ativo, considerando os sete dias anteriores. }\end{array}$ & Todos & $\begin{array}{l}73,9^{\mathrm{a}} \\
52,5^{\mathrm{b}}\end{array}$ & NA \\
\hline $\mathrm{Gama}^{16}$ & Praticar $<60$ minutos de atividade física por dia. & Todos & $\begin{array}{l}38,2^{\mathrm{c}} \\
18,5^{\mathrm{d}} \\
21,5^{\mathrm{e}}\end{array}$ & NA \\
\hline Mazaro $^{17}$ & Não realiza atividade física & NA & 72,9 & NA \\
\hline
\end{tabular}

$\mathrm{NA}=$ Não apresenta; $\mathrm{M}$ = Masculino; $\mathrm{F}$ = Feminino; ${ }^{*}$ Prevalência geral; ${ }^{* *}$ Prevalência por sexo; Todos = Lazer, deslocamento, atividades ocupacionais e domésticas; $\mathrm{a}=$ crianças com sobrepeso; $\mathrm{b}=$ crianças com peso normal; $\mathrm{c}=$ baseline crianças pré-púberes; $\mathrm{d}=$ follow-up crianças pré-púberes; $\mathrm{e}=$ follow-up crianças púberes

No Quadro 1 são apresentadas as informaçóes dos critérios metodológicos empregados. Na maioria dos estudos o questionário foi utilizado, sendo que em um deles foi utilizado um recordatório ${ }^{11}$. Em relação ao processo de amostragem e procedimentos de seleçáo, em três estudos foi empregada a seleção por conveniência ${ }^{11,15,16}$. 
Quadro 1: Características dos estudos com crianças brasileiras de acordo com os critérios metodológicos empregados.

\begin{tabular}{|c|c|c|}
\hline $1^{\mathbf{0}}$ Autor & Instrumento & Amostragem e procedimentos de seleçáo \\
\hline Giugliano $^{11}$ & Recordatório & Seleção das crianças por conveniência \\
\hline Assis $^{12}$ & Questionário & Todas as crianças das escolas que foram randomicamente selecionadas, de acordo com a área geográfica \\
\hline Baruki $^{13}$ & Questionário & Escolas sorteadas de acordo com a área geográfica, e as turmas de alunos sorteadas (conglomerados) \\
\hline Gama $^{14}$ & Questionário & Aleatória sistemática \\
\hline Siqueira $^{15}$ & Questionário & Seleção das crianças por conveniência \\
\hline Gama $^{16}$ & Questionário & Seleção das crianças por conveniência \\
\hline Mazaro $^{17}$ & Questionário & Escolas sorteadas de acordo com a área geográfica, e alunos sorteados probabilisticamente \\
\hline
\end{tabular}

NA = Não apresenta.

\section{Discussão}

Nesse estudo, a prevalência de inatividade física variou de 14,2 a 73,9\%. Em revisão sobre atividade física de adolescentes brasileiros, Tassitano et al. ${ }^{3}$ encontraram variação de 39 a 93,5\% de adolescentes com baixo nível de atividade física. Nota-se que nesse estudo ${ }^{3}$ a amplitude superior da variação de prevalência de inatividade física foi maior do que o observado na presente revisão. Presume-se, portanto, que os adolescentes são mais inativos em relação às crianças brasileiras.

No estudo de coorte de Gama et al. ${ }^{16}$, foram verificadas proporções de crianças fisicamente inativas de 38,2\% no baseline, e 18,5 e 21,5\% no follow-up, em crianças pré-púberes e púberes, respectivamente. No entanto, os autores não apresentam os motivos pelo decréscimo entre os estudos. Possivelmente, a discrepância entre as proporçóes apresentadas em cada estudo esteja relacionada ao acréscimo no quantitativo de crianças na segunda pesquisa, tendo em vista o procedimento de coorte aberta. É importante destacar que em estudo conduzido com informaçóes de diferentes países sobre os níveis de atividade física, foi estimado que $80 \%$ dos adolescentes, sendo $53 \%$ do sexo masculino e $95 \%$ do sexo feminino, não apresentavam 60 minutos diários de atividade física ${ }^{18}$, o que aponta a necessidade de promovê-las nessa fase da vida ${ }^{10}$.

Nesse estudo foi observado um baixo número de publicaçóes sobre atividade/inatividade física em crianças brasileiras. A quantidade de publicaçôes que compuseram esta revisão difere de outros estudos relacionados a esse tema no Brasil ${ }^{7,9}$. Nos achados de Sousa ${ }^{9}$, no período de 1999 a 2010 foi observado aumento da quantidade de publicaçóes sobre inatividade física em estudantes universitários, especialmente a partir de 2006. Em outra revisão sobre a atividade física no Brasil, os autores observaram crescimento significativo a partir de 2000, entre as publicaçóes de 1990 a $2005^{7}$. A realização de mais pesquisas sobre inatividade física, com nível de excelência, poderá favorecer o esclarecimento de informaçóes sobre o presente tema em crianças brasileiras. Dumith ${ }^{8}$ atribui o número baixo de publicaçóes com crianças em virtude das dificuldades logísticas para a coleta de dados nessa faixa etária. De maneira específica, isso acontece pelo retorno de questionários preenchidos, do viés de resposta, ou do não conhecimento de informaçóes relevantes para o estudo.

Quanto ao delineamento dos estudos, o tipo transversal foi verificado como principal. Outras revisóes corroboram os resultados desse estudo ${ }^{3,8,9}$, refletindo escassez de outros tipos de investigação. Em Sousa ${ }^{9}$ e de Dumith $^{8}$, por exemplo, todas as pesquisas apresentaram delineamento transversal. Já Tassitano et al. ${ }^{3}$, em revisão sobre a atividade física em adolescentes brasileiros, identificaram que dois trabalhos apresentaram delineamento de casos e controles, sendo os demais de delineamento transversal. Embora esse tipo seja o mais empregado nos estudos que fizeram parte dessa revisão, ele apresenta limitações importantes, como a dificuldade para investigar condiçóes de baixa prevalência, já que isso implicaria num maior tamanho amostral ${ }^{19}$. Além disso, os estudos transversais são desenvolvidos com casos prevalentes, e a coleta de dados da exposição, junto com seu desfecho, se desenvolvem em um único momento, dificultando o estabelecimento de relaçóes de causalidade ${ }^{19}$.

De forma semelhante à presente revisão, em outros estudos foram identificadas desigualdades quanto às regiōes pesquisadas ${ }^{8,9}$. Hallal et al..$^{7}$ observaram disparidade regional nas publicaçôes sobre a atividade física no Brasil. A maioria dos estudos identificados pelos autores foram realizados nas regióes Sudeste (43\%) e sul (36\%). $\mathrm{Na}$ revisão sistemática realizada por Dumith ${ }^{8}$, observou-se o predomínio das publicações na região Sul do país. Sousa ${ }^{9}$ identificou que os estudos sobre inatividade física em universitários brasileiros foram predominantes nas regióes Nordeste e Sudeste. A maior potencialidade das regióes Sul e Sudeste com a produção de pesquisas se pauta, parcialmente, pela disponibilidade superior de programas de pós-graduação, responsáveis pelo desenvolvimento das pesquisas ${ }^{20}$.

Foi observada a unanimidade quanto ao uso de questionários para obtenção de dados nos estudos que compuseram esta revisão, exceto por um que utilizou o 
recordatório das atividades físicas diárias ${ }^{11}$. Em apenas um estudo foi apresentado melhor detalhamento sobre o questionário empregado para obtenção das informaçôes, que apresentam níveis satisfatórios de aplicabilidade $^{15}$. No entanto, apesar das vantagens no uso de questionários para mensurar o nível de atividade física, eles são instrumentos extremamente subjetivos, e necessitam da capacidade do sujeito da pesquisa em recordar as atividades realizadas durante o período de referência do estudo (por exemplo: últimos sete dias; mês anterior), que em crianças pode aumentar a margem de erro na medida da variável ${ }^{21}$. Além disso, o emprego de um instrumento adaptado sem testagem adequada das capacidades psicométricas pode minimizar a validade das informaçôes ${ }^{21}$. A carência de métodos diretos e fidedignos para mensurar o nível de atividade física, como o uso de pedômetros, sensor de movimento e acelerômetros, limita a obtenção de resultados precisos ${ }^{21}$.

Os estudos apresentaram distintas definiçóes operacionais da inatividade física ou seu baixo nível, tais como: não realizar atividade física ${ }^{17}$; a percepção dos responsáveis em relação à prática das crianças ${ }^{12}$; ou não atender a recomendaçóes dos níveis de atividade físi$\mathrm{ca}^{14,16}$. A utilização de diferentes definiçóes, com intuito de classificar os níveis de atividade física de crianças, compromete principalmente a comparabilidade entre os estudos. Além disso, classificar crianças com base em critérios que não avaliam a adequação do grupo investigado com as recomendaçóes de atividade física ${ }^{2,10}$ pode conduzir ao erro de avaliação e, assim, inviabilizar as possíveis tomadas de decisão, como programas e projetos para o estímulo da prática.

Sobre as limitações do presente estudo, aponta-se a exclusão de dissertaçóes, teses, monografias, dentre outros tipos de publicaçôes, que pode comprometer a quantidade de informaçôes sobre a inatividade física em crianças. Além disso, destaca-se a consulta a somente uma base de dados, a Bireme, o que inviabiliza a obtenção de outras publicaçôes em periódicos nacionais não indexados nessa base, ou estudos publicados em periódicos internacionais. Entretanto, a Bireme é uma base de dados que incorpora outras bases como LILACS, PubMed e SciELO. Apesar de tais limitações, acredita-se que essa revisão sistematizou as principais publicaçôes sobre inatividade física em crianças brasileiras.

\section{Conclusões}

Esse trabalho de revisão sistemática evidenciou elevada prevalência de inatividade física em crianças de 5 a 11 anos de idade, tendo como principal instrumento o questionário, com distintas formas de definição operacional de inatividade física. Outro aspecto destacado foi o tipo de estudo utilizado nas pesquisas, com ênfase no método transversal, e a predominância de publicaçóes provenientes da regiáo Sudeste.

É importante caracterizar a relação entre atividade física e promoção da saúde para as pessoas de todas as faixas etárias, e os achados dessa revisão sistemática levam à reflexão sobre a relevância dada às investigaçóes desse problema em crianças. Para tanto, a promoção de programas de estímulo e orientaçáo, ou projetos com profissionais capacitados, por exemplo, nas escolas, que envolvam as crianças e seus pais, tornam-se fundamentais para o aumento dos níveis de atividades físicas e a possível diminuição de doenças crônicas não transmissíveis em outras fases da vida.

\section{Referências}

1. Oehlschlaeger MHK, et al. Prevalência e fatores associados ao sedentarismo em adolescentes de área urbana. Rev de Saúde Púb. 2004;38(2):157-63.

2. Strong WB, et al. Evidence based physical activity for school-age youth. The Journal of Pediatrics. 2005;146(6):732-7.

3. Tassitano RM, et al. Atividade física em adolescentes brasileiros: uma revisão sistemática. Rev Bras de Cineantropometria e Desempenho Humano. 2007;9(1):55-60.

4. Molina MCB, et al. Fatores de risco cardiovascular em crianças de 7 a 10 anos de área urbana, Vitória, Espírito Santo, Brasil. Cader de Saúde Púb. 2010;26(5):909-17.

5. Costa FF, Assis MAA. Nível de atividade física e comportamentos sedentários de escolares de sete a dez anos de Florianópolis-SC. Rev Bras de Atividade Física e Saúde. 2011;16(1):48-54.

6. Schimidt MI, et al. Chronic non-communicable diseases in Brazil: burden and current challenges. Lancet. 2011;377(9781):1949-61.

7. Hallal PC, et al. Evolução da pesquisa epidemiológica em atividade física no Brasil: revisão sistemática. Rev de Saúde Púb. 2007;41(3):453-60.

8. Dumith SC, et al. Atividade física no Brasil: uma revisão sistemática. Cader de Saúde Púb. 2009;25(3):415-26.

9. Sousa TF. Inatividade física em universitários brasileiros: uma revisão sistemática. Rev Bras de Ciências da Saúde. 2011;9(29):47-55.

10. United States Department of Health and Human Services (USDHHS). Physical Activity Guidelines for Americans. Washington, 2008 [acesso em 17 set 2015]. Disponível em: http://www.health.gov/paguidelines/pdf/paguide.pdf

11. Giugliano R, Carneiro EC. Fatores associados à obesidade em escolares. Jornal de Pediatria. 2004;80(1):17-22.

12. Assis MAA, et al. Overweight and thinness in 7-9 year old children from Florianópolis, Southern Brazil: a comparison with a French study using a similar protocol. Rev de Nutrição. 2006;19(3):299-308. 
13. Baruki SBS, et al. Associação entre estado nutricional e atividade física em escolares da Rede Municipal de Ensino em Corumbá-MS. Rev Bras de Medicina do Esporte. 2006;12(2):90-4.

14. Gama SR, Carvalho MS, Chaves CRMM. Prevalência em crianças de fatores de risco para as doenças cardiovasculares. Cader de Saúde Púb. 2007;23(9):2239-45.

15. Siqueira PP, Alves JGB, Figueiroa JN. Fatores associados ao excesso de peso em crianças de uma favela do nordeste brasileiro. Rev Paulista Pediatria. 2009;27(3):251-7.

16. Gama SR, et al. Cohort study for monitoring cardiovascular risk factors in children using a primary health care service: methods and initial results. Cader de Saúde Púb. 2011;27(3):510-20.
17. Mazaro IAR, et al. Obesidade e fatores de risco cardiovascular em estudantes de Sorocaba, SP. Rev da Associação Medica Bras. 2011;57(6):674-80.

18. Hallal PC, et al. Global physical activity levels: surveillance progress, pitfalls, and prospects. Lancet. 2012;380(9838):247-57.

19. Bastos JLD, Duquia RP. Um dos delineamentos mais empregados em epidemiologia: estudo transversal. Scien Médica. 2007;17(4):229-32.

20. Coordenação de Aperfeiçoamento de Pessoal de Nível Superior (CAPES) [acesso em 17 set 2015]. Disponível em: http://www.capes.gov.br/

21. Barros MVG, Nahas MV. Medidas da atividade física. Londrina, PR: Midiograf; 2003. 